\title{
Stochastic short-term maintenance scheduling of GENCOs in an oligopolistic electricity market
}

\author{
Mohammad Ali Fotouhi Ghazvini, Bruno Canizes, Zita Vale, Hugo Morais
}

\author{
A B S T R A C T
}

In the proposed model, the independent system operator (ISO) provides the opportunity for maintenance outage rescheduling of generating units before each short-term (ST) time interval. Long-term (LT) scheduling for 1 or 2 years in advance is essential for the ISO and the generation companies (GENCOs) to decide their LT strategies; however, it is not possible to be exactly followed and requires slight adjustments. The Cournot-Nash equilibrium is used to characterize the decision-making procedure of an individual GENCO for ST intervals considering the effective coordination with LT plans. Random inputs, such as parameters of the demand function of loads, hourly demand during the following ST time interval and the expected generation pattern of the rivals, are included as scenarios in the stochastic mixed integer program defined to model the payoff-maximizing objective of a GENCO. Scenario reduction algorithms are used to deal with the computational burden. Two reliability test systems were chosen to illustrate the effectiveness of the proposed model for the ST decision-making process for future planned outages from the point of view of a GENCO.

Keywords:

Cournot model

Game theory

Maintenance scheduling

Nash equilibrium

Oligopolistic market

Stochastic mixed integer programming

\section{Introduction}

\subsection{Background and aim}

Generation maintenance scheduling of large-scale power systems is a complex multi-objective optimization problem. In centralized power systems, the system operator schedules and finalizes the outage plans for LT and ST time horizons, and the generating units mainly follow the plans imposed by the operator. By contrast, the maintenance scheduling in restructured power systems mainly seek the objectives of the generating units and the companies that manage and own these units, and the operator has to keep the security and reliability indices at the standard level.

LT maintenance outage scheduling, which maximizes the payoff over a yearly or longer time horizon, is almost unavoidable prior to any ST planning [1]. The obvious difference between LT and ST scheduling is the time horizon and the time increment. In LT maintenance scheduling, the shortest maintenance outage time scale is chosen with the accuracy of a day or several days for 1-2 years [2]. The ST outage scheduling is done with the accuracy of an hour or less and needs coordination with LT planning results by accepting constraints derived from LT schedules [1]. However, the LT schedule is not totally fixed and can slightly change to satisfy the ST scheduling requirements [2].

The system planners face more uncertainty during LT operational planning of power systems in comparison to ST planning. Although uncertainty remains in various parameters during ST scheduling, it is vital for the efficient and secure operation of the system. The GENCOs are usually asked to submit the proposals for maintenance outages of their units several times during a year, and each time for a specific interval determined by the ISO. GENCOs run a unit commitment with the objective of maximizing the total payoff, while the ISO chooses the objectives that maintain the security and reliability criteria of the system [3]. In ST scheduling, the GENCOs have more knowledge about their rivals' decisions 


\section{Nomenclature}

Indices
$s$
$t$
$i$
$j$

Variables

$\rho \quad$ generation of a unit (MW)

$\rho_{i} \quad$ total generation offered to a market by GENCO $i$ (MW)

$\rho_{-i} \quad$ total generation offered to a market by all GENCOs except GENCO $i(\mathrm{MW})$, based on LT maintenance plans

$x \quad$ binary decision variable for maintenance status, 1 if the unit is offline for maintenance and 0 otherwise

$z \quad$ binary decision variable for shutdown, 1 if the unit shuts down in the beginning of period $t$ and 0 otherwise binary decision variable for online status, 1 if the unit is online during period $t$ and 0 otherwise

binary decision variable for startup, 1 if the unit starts up at the beginning of period $t$ and 0 otherwise probability of occurrence for scenario $s$ intercept of the system inverse linear demand function $(€ / \mathrm{MW} \mathrm{h})$ $b \quad$ slope of the system inverse linear demand function $(€)$ $\left.(\mathrm{MW} \mathrm{h})^{2}\right)$ demand (MW)

$R \quad$ reserve (MW)

\section{Parameters}

$h_{t} \quad$ number of hours in time period $t$

$M_{i} \quad$ maximum number of units simultaneously in outage for maintenance

$\overline{\mathrm{P}}, \underline{\mathrm{P}} \quad$ maximum/minimum generation capacity (MW)

$N_{i j} \quad$ number of time periods required for the maintenance outage of generating unit $j$ of GENCO $i$

$T$ number of time periods

$C^{S D} \quad$ shutdown cost of a unit $(€)$

$C^{\mathrm{SU}} \quad$ startup cost of a unit $(€)$

$C^{\mathrm{M}} \quad$ maintenance cost of a unit $(€ / \mathrm{MW} / \mathrm{h})$

$C^{\mathrm{F}} \quad$ fixed cost of a unit $(€ / \mathrm{h})$

$C^{\mathrm{P}} \quad$ production cost of a unit $(€ / \mathrm{MW})$

Sets

$\Delta_{i} \quad$ generating units owned by GENCO $i$ through publicly available information released by the ISO [1]. This information was not available when the GENCOs and the ISO were planning for a LT time horizon. The new knowledge certainly affects the decision-making of individual GENCOs; they might prefer to alter their previously planned schedules to achieve higher payoffs according to the new information they have.

A common characteristic in most of the deregulated electricity markets is their oligopoly structure which is caused by the limited number of competitors in the market [4]. In comparison to monopoly and perfect competition markets, where the agents' interactions are simpler, game-theoretic approaches are used to model the interactions between the GENCOs in an oligopolistic electricity market.

The proposed model serves as a decision support tool for GENCOs during ST maintenance outage scheduling in an oligopolistic electricity market. It considers the decisive influence of rivals on the outage decisions of each individual GENCO. The maintenance plans developed by GENCOs through this procedure act as proposals for the ISO, and accordingly require approval before implementation.

\subsection{Literature review and contribution}

Coordination between LT generation scheduling and ST security-constrained generation scheduling with the presence of network constraints is discussed in [2,3]. All the decision-making process is modeled and analyzed from the viewpoint of a system operator in centralized electricity markets. A comprehensive review over the maintenance scheduling problems in the generation and transmission sector of restructured power systems with their solutions is presented in [3]. An iterative approach for LT maintenance scheduling is presented in [5] that guarantees a satisfactory level of system reliability in a manner acceptable to all participating GENCOs and the ISO. In [6] maintenance scheduling is solved by the system operator based on the plans that the producers have submitted with the respective penalties they accept to pay if their desired plan is selected. The Cournot model applied in [7] for maintenance scheduling of the generating units is based on the fact that each GENCO can estimate the capacity and cost parameters of the competitor GENCOs. The merit of considering Cournot model instead of conventional price-based revenue functions in ST decision-making of virtual power players is discussed in [8].

In [1] the participating GENCOs in a single round auction, besides their own generation portfolio, have sufficient knowledge about other participating generating units during generation bidding process. The work in [4], introduces an evolutionary game for characterizing the interactions in a electricity market, and implements a competitive coevolutionary approach to determine the Nash-Cournot equilibrium of an oligopolistic electricity market. The effects of generator availability and demand uncertainty on electricity prices under Cournot competition is modeled in [9]. Cournot model is employed to characterize strategic bidding for the spot market and modeling the forward market [10]. In [11] optimal control is applied to study generator bidding in an oligopolistic electricity market, and the repeated bidding process in real-time markets are modeled as dynamic feedback systems. A consumer based price elasticity approach for load reduction in order to study the demand response actions in distribution networks is introduced in [12].

The work in [13] presents a stochastic model for scheduling the GENCOs' maintenance outages from the viewpoint of a GENCO, and requires the approval of the ISO before implementation. A GENCOs' mid-term generation scheduling in volatile operating conditions with large integration of intermittent resources is studied in [14]. It also applies the market price forecasts in the risk-constrained stochastic price-base unit commitment [14]. Failure and repair rates of the units are used in [15] to solve a unit commitment problem considering the probabilistic unit outages. Each generated scenario represents a series of randomly generated availability and unavailability periods of time for each unit [15]. The stochastic programming approach introduced in [16] optimally solves the electricity procurement problem faced by large consumers. The uncertain trading of wind energy in a market environment is the main constraint of the stochastic programming approach proposed in [17]. The stochastic model introduced in [18] is designed to ensure the ISO that the system will have secure operation during the scheduling horizon. The same methodology has been implemented in [19] with detailed representation of high voltage DC transmission system. The aim of [20] is to prove that applying a quantified method while dealing with intermittent resources increases the reliability 
despite the inherent uncertainties and risks of the system, and the results show the advantages of stochastic unit commitment over deterministic approaches in power systems with large integration of wind units. A neural network based technique is used in [21] for modeling the uncertainty of the ST intermittent resources generation prediction.

The model presented in this paper completes the coordination procedure of [6] by introducing an appropriate approach for GENCOs to achieve the determinate number of outage plans for the ST period. In this model, the GENCOs have sufficient knowledge about what the competing GENCOs might decide in a future ST horizon, and the uncertainty of stochastic input data for the target GENCO in an oligopolistic electricity market is incorporated in the model.

\subsection{Paper organization}

The rest of the paper is organized as follows: Section 2 is devoted to the assumptions that our model is based on. In Section 3 , the stochastic optimization formulation based on the Cournot model in an oligopolistic electricity market is introduced. Section 4 presents case studies and discusses numerical results. Finally, in Section 5, some relevant conclusions are accordingly made and the possible future extensions of the proposed model are suggested.

\section{Model assumptions}

GENCOs as self-interested entities are in charge of managing and scheduling the outage plans of their own units [13]. On the other hand, the ISO has the responsibility to make the final outage decision for the generating units' maintenance considering the reliability and security constraints of the system. It also has to take into account the plans submitted by each GENCO. The proposed model is based on the following assumptions

- When the problem is studied from the point of view of a GENCO which intends to obtain the ST outage proposals in order to send to the ISO, the optimization of generation bids and maintenance outages must take into consideration the behavior of the competing GENCOs and should be coordinated with LT planning results [1]. Each GENCO seeks to come to a realization of the generation patterns that other rivals will have during the next time horizon and the maintenance outage plans that they are going to suggest to the ISO.

- The main information source to build up their image of others' most probable generation and maintenance strategy is based on what they derive from LT maintenance plans determined for 1 or 2 years time horizon. However, the rivals also have the opportunity to deviate from the path that was finalized for the LT time horizon, accepting the limitations that are imposed by the ISO. In this model, the main limitation that is imposed by the ISO is giving the opportunity of maintenance outage only to those units that were considered for maintenance during the following ST time horizon in the LT plans released earlier.

- The GENCOs' ability in predicting the future behavior of their rival GENCOs is modeled in the oligopolistic electricity market structure, where few competing GENCOs are acting strategically to maximize their profit [4]. The consumers are assumed to have no strategic behavior in the market, and can only affect the price by their inverse demand function [4].

- Uncertainties in several input parameters, such as the hourly demand, demand function of the loads and the estimation of the rivals' generation pattern during the ST period are modeled. Uncertainty of production for the self-scheduling GENCO due to the intermittent nature of the resources is not considered in the proposed model.

\section{Stochastic model for oligopoly competition}

Planned outages of the generating units and the transmission lines are necessary for preventive maintenance which has significant effect on the efficiency and ensures the secure operation of the units and of the whole system. In outage scheduling various constraints of the generating units regarding to their technical, cost and the crew limitations are imposed to the problem. Optimal scheduling of energy resources is a critical issue for all market participants such as the GENCOs and the ISO, and the outage scheduling is the most influential decision that affects the optimal operation of power systems. The GENCOs seek their profit maximization, and the ISO schedules the system aiming at the minimum cost while satisfying the system reliability and security constraints.

Scheduling in electrical energy systems very much depends on forecasted and deterministic input data. It is necessary to investigate the effect of forecast errors and contingencies in LT and ST scheduling. The level of uncertainty and the type of data differ when the problem is being solved from the viewpoint of different market participants; for instance the consumers and the producers do not face similar input data for their decision support system while bidding in the market. The competition model of an electricity market with few competing GENCOs is introduced first, and the mechanism that an individual GENCO implements to determine the outage plans in this environment is also characterized. The stochastic model that each GENCO uses and the way that it can deal with uncertainties is given next.

\subsection{Oligopoly competition model}

In the deregulated electricity markets based on the oligopoly structure, the electricity price is not determined by a regulatory authority; the market demand, supply conditions, and the strategic behavior of GENCOs are the main influential factors [9]. In oligopolistic electricity markets the decisions of GENCOs are interconnected, and each GENCO is big enough to influence the electricity price [7]. Each GENCO maximizes its total payoff by changing the decision variable of production at each time period [10]. The Cournot model, a game theory-based framework, is applied to address the strategic interaction between GENCOs in an oligopolistic electricity market and to analyze the Nash equilibrium [11].

Nonlinear demand functions usually yield to problems that are difficult to solve; therefore, a linear demand function (1) is selected to show the relationship between electricity price and the demand [9]

$Q=\lambda-\gamma \cdot P$.

In Eq. (1), $\gamma \geqslant 0$, and $\lambda$ shows the demand intercept. The variable $Q$ represents the actual demand and $P$ is the electricity price at time period $t$ [9]. In the proposed model, GENCO $i$ submits its maintenance schedule simultaneously with other GENCOs. Eq. (2) shows the relationship between the production of GENCOs and the total demand at period $t$. GENCO $i$ chooses the payoff-maximizing quantity of generation in the belief that other GENCOs have fixed production decisions [9]

$\rho_{i}+\rho_{-i}=Q$.

In the proposed maintenance scheduling model, the inverse linear demand function (3), is used to include the price of electricity in the objective function, while optimizing the payoff from the perspective of GENCO $i$. Inverse demand function maps the amount of electricity demanded to the market price which is a dependent variable [22]. Eq. (4) shows how the parameters of the inverse linear demand function are calculated from the parameters in linear demand function 
$P=a-b \cdot Q$

$a=\lambda / \gamma, \quad b=1 / \gamma$.

Unlike the demand curve used in [23], with constant elasticity at all different prices and quantities, the price elasticity changes for different values of demand in the inverse linear demand function. The price elasticity of demand (PED) is defined as the ratio of the percentage change in quantity to the percentage change in price (5). PED shows the demand response of a good or service to changes in its price [12]

$\mathrm{PED}=\frac{\Delta Q / Q}{\Delta P / P}$

\subsection{Stochastic model}

Mathematical programming models provide an appropriate framework to address maintenance outage decisions in a precise manner. Maintenance outage scheduling of generating units is typically of integer nature while the power generation decisions are continuous [24]. The complexity of planning for maintenance outage from the system operator point of view is considerable in comparison to when the GENCOs are addressing the problem. The main reason for this added complexity is the inherent responsibility of system operators to guarantee the security and reliability of the system [6].

In the proposed model, prior to implementing the LT maintenance decisions, the ISO asks those generating units that were planned for maintenance outage during the considered ST time interval to submit the new proposals. The ST time interval varies between one to several weeks. Some GENCOs might be interested to submit several plans with different levels of priority; consequently, they should expect penalties for the plans that deteriorate the system security or reliability constraints.

Different degrees of uncertainty are seen in most of the decision-making problems faced by electricity market players [25]. Even with the lack of prefect information and with regard to time limits that they face, the decisions should be made several time periods ahead of the scheduling time horizon. In this paper, we address the problem of finding optimal ST generation policies for a specific GENCO in a non-cooperative oligopoly, taking into account the system uncertainties.

The ultimate objective of a GENCO in deregulated electricity markets is to maximize the difference between revenues from generating for the pool and the generation cost (6). The term $\Pi_{i}$ in (6) refers to the total payoff of GENCO $i$ during the scheduling horizon. A scenario-variable formulation is used to consider the uncertainty of several parameters [25]. This objective function is the expected payoff computed by the summation of the payoff in each scenario multiplied by its probability [25]. It is assumed that GENCO $i$ has estimates of other GENCOs' production during each period via the LT plans that have been released by the ISO to all market players. Time increments are included in the model by considering $h_{t}$ in the objective function and the constraints. For instance, 0.25 is assigned to $h_{t}$ for all time periods if time increments of $15 \mathrm{~min}$ are assumed

$$
\begin{aligned}
\operatorname{Max} \Pi_{i}= & \sum_{s} p^{s} \cdot\left\{\sum _ { t = 1 } ^ { T } \left\{\left[a^{s}(t)-b^{s}(t) \cdot D^{s}(t) \cdot h_{t}\right] \cdot \rho_{i}^{s}(t) \cdot h_{t}\right.\right. \\
& -\sum_{j \in \Delta_{i}}\left(z_{i j}^{S}(t) \cdot C_{i j}^{S \mathrm{D}}+y_{i j}^{s}(t) \cdot C_{i j}^{\mathrm{SU}}+h_{t} \cdot x_{i j}^{s}(t) \cdot C_{i j}^{\mathrm{M}} \cdot \overline{\mathrm{P}_{i j}}+h_{t}\right. \\
& \left.\left.\left.\cdot\left(C_{i j}^{\mathrm{F}} \cdot u_{i j}^{\mathrm{s}}(t)+C_{i j}^{\mathrm{P}} \cdot \rho_{i j}^{s}(t)\right)\right)\right\}\right\} .
\end{aligned}
$$

In the proposed model, the electricity producer faces uncertainty in demand, the parameters of the demand function and expectations that it has of other producers' generation strategies during the planning time horizon. Equation (7) shows that the generation of GENCO $i$ at each period equals the total power that all the generating units owned by the GENCO produce in that period. Constraint (8) guarantees a minimum reserve for each time period

$\sum_{j \in G_{i}} \rho_{i j}^{s}(t)=\rho_{i}^{s}(t), \quad \forall i, t, s$.

$\rho_{i}^{s}(t)+\rho_{-i}^{s}(t)=D^{s}(t)+R^{S}(t), \quad \forall i, t, s$.

Constraint (9) shows that each generating unit should be maintained for a specified number of time periods. Constraint (10) ensures the continuity of maintenance in generating units [5]

$\sum_{t=1}^{T} X_{i j}^{s}(t)=N_{i j}, \quad \forall s, i, \forall j \in \Delta_{i}$

$x_{i j}^{s}(t)-x_{i j}^{s}(t-1) \leqslant x_{i j}^{s}\left(t+N_{i j}-1\right), \quad \forall i, t, s, \forall j \in \Delta_{i}$

Constraint (11) expresses that a unit which is on outage for maintenance will not deliver energy, and consequently cannot be committed [13]

$x_{i j}^{s}(t)+u_{i j}^{s}(t) \leqslant 1, \quad \forall i, t, s, \forall j \in \Delta_{i}$.

The output of each generating unit should be within a range limited by its minimum and maximum generation capacity (12) [13]

$u_{i j}^{s}(t) \cdot \underline{\mathrm{P}_{i j}} \leqslant \rho_{i j}^{s}(t) \leqslant u_{i j}^{s}(t) \cdot \overline{\mathrm{P}_{i j}}, \quad \forall i, t, s, \forall j \in \Delta_{i}$.

The logic of startup and shutdown is defined using binary decision variables for the online, startup, and shutdown status as shown in constraints (13) and (14). It is necessary to have the initial state of the binary decision variables for online status as an input

$y_{i j}^{s}(t)-z_{i j}^{s}(t)=u_{i j}^{s}(t)-u_{i j}^{s}(t-1), \quad \forall i, t, s, \forall j \in \Delta_{i}$.

$y_{i j}^{s}(t)+z_{i j}^{s}(t) \leqslant 1, \quad \forall i, t, s, \forall j \in \Delta_{i}$.

The limitations of the maintenance crew and the equipments of a GENCO are restraints on the maximum number of units simultaneously in outage (15)

$\sum_{j \in \Delta_{i}} x_{i j}^{s}(t) \leqslant M_{i}, \quad \forall i, t, s$

In stochastic optimization of the total payoff, reaching a decision that guarantees a larger mean for payoff and a smaller variance are always sought by the owners of the firms. This indicates that the expected value of the payoff is high and the probability of obtaining payoff values different from the expected payoff is low [25].

Defining the problem in a stochastic structure adds to the complexity of the problem due to the increase in the number of variables; therefore, it would be convincing to use a measure to show the advantages of this representation. The value of the stochastic solution (VSS) is a measure used to show the benefits of using stochastic representation for the problem in comparison to a simpler deterministic structure [25]. Equation (16) shows that subtracting the optimal objective function value of the modified stochastic problem $\Pi^{\mathrm{D} *}$ from the stochastic programming solution $\Pi^{S *}$ gives the VSS for a maximization problem [25]

$\mathrm{VSS}_{\mathrm{MAX}}=\Pi^{\mathrm{S} *}-\Pi^{\mathrm{D} *}$ 


\subsection{Scenario generation}

The GENCO that intends to submit the maintenance outage plans of the following ST period to the ISO, creates several scenarios to face the future random disturbances, such as load forecasts, price elasticity of the loads and the generation pattern of the competing GENCOs [18]. In the scenario-based optimization model, the objective of the GENCO is to maximize the weighted-average profit [18]. The probability of occurrence for each scenario, which is a possible state of uncertain circumstances, is shown by the weight assigned to it. Solving continuous stochastic optimization problems is very difficult, or even impossible. Thus representing the continuous random variables with discrete structure in actual decision-making process is indispensable [25].

The vector $R^{s}$ in (17) shows the system state in scenario $s$ and is composed of three vectors $r_{1}^{s}, r_{2}^{s}$ and $r_{3}^{s}$ which are respectively assigned to the parameters of the inverse linear demand function, hourly load of the ST time horizon, and the generation pattern of rival GENCOs

$R^{s}=\left[r_{1}^{s}, r_{2}^{s}, r_{3}^{s}\right]$

Constructing the demand functions by applying statistical techniques such as regression analysis to historical data is not the concern of this paper. In order to model the stochastic behavior of loads in reaction to price changes, the historical price elasticity of demand at the average electricity price over a specific time horizon is analyzed to find the points with higher density and the weights calculated for each point.

It is assumed that the probability distribution function of load forecast error is available. In other words, a standard probability distribution has been chosen and the parameters are adjusted to fit the data. Load uncertainty, which is one of the most influencing parameters of system uncertainty, is based on load forecast errors [26]. Discretization of the continues distribution function is carried out based on the technique proposed in [26] which gives the probabilities of $n$ load levels (Fig. 1). Roulette wheel selection mechanism is used to generate the scenarios for each hour. Generated random numbers between 0 and 1 fall in the normalized probability range of the $n$ defined levels in the roulette wheel [26].

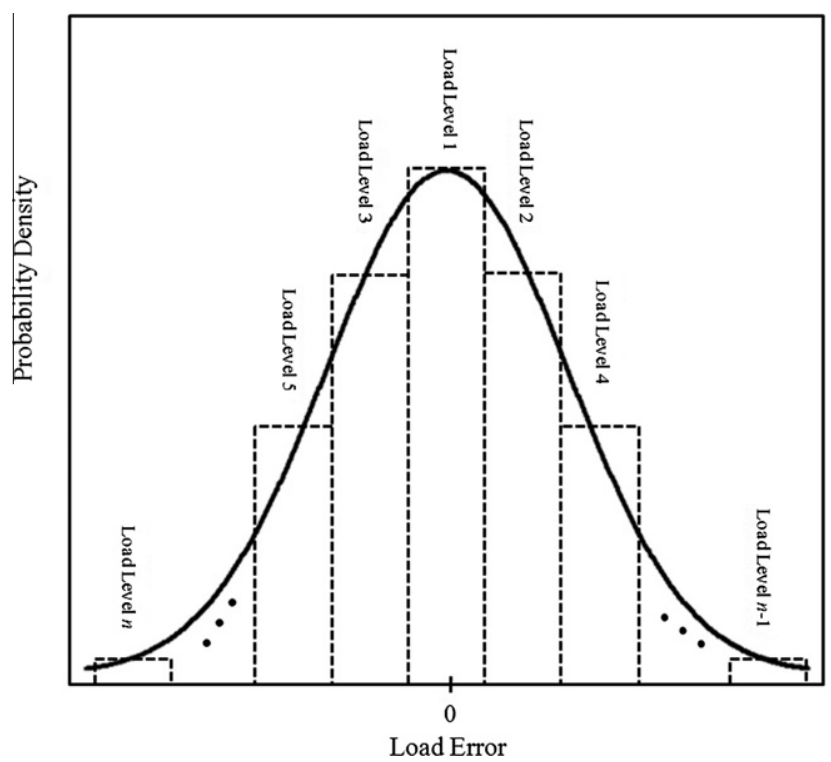

Fig. 1. Discretization of the load forecast error probability distribution function (adapted from [26]).
Table 1

Generating units data (case 1).

\begin{tabular}{|c|c|c|c|c|c|}
\hline $\begin{array}{l}\text { Unit } \\
\text { no. }\end{array}$ & $\begin{array}{l}\text { Capacity } \\
\text { (MW) }\end{array}$ & $\begin{array}{l}\text { Minimum } \\
\text { power } \\
(\mathrm{MW})\end{array}$ & $\begin{array}{l}\text { Repair } \\
\text { rate }\end{array}$ & $\begin{array}{l}\text { Failure } \\
\text { rate }\end{array}$ & $\begin{array}{l}\text { GENCO } \\
\text { no. }\end{array}$ \\
\hline 1 & 12 & 2.40 & 0.94652 & 0.03742 & 2 \\
\hline 2 & 12 & 2.40 & 0.88966 & 0.05736 & 2 \\
\hline 3 & 12 & 2.40 & 0.96426 & 0.01775 & 4 \\
\hline 4 & 12 & 2.40 & 0.97204 & 0.05772 & 1 \\
\hline 5 & 12 & 2.40 & 0.94863 & 0.01851 & 4 \\
\hline 6 & 20 & 4.00 & 0.95588 & 0.05043 & 4 \\
\hline 7 & 20 & 4.00 & 0.95454 & 0.05485 & 2 \\
\hline 8 & 20 & 4.00 & 0.93654 & 0.01702 & 1 \\
\hline 9 & 20 & 4.00 & 0.97420 & 0.05522 & 4 \\
\hline 10 & 76 & 15.20 & 0.89939 & 0.04271 & 4 \\
\hline 11 & 76 & 15.20 & 0.97488 & 0.01259 & 2 \\
\hline 12 & 76 & 15.20 & 0.90084 & 0.05210 & 3 \\
\hline 13 & 76 & 15.20 & 0.96110 & 0.05622 & 1 \\
\hline 14 & 100 & 25.00 & 0.96946 & 0.04382 & 3 \\
\hline 15 & 100 & 25.00 & 0.89803 & 0.04766 & 2 \\
\hline 16 & 100 & 25.00 & 0.97015 & 0.04695 & 3 \\
\hline 17 & 100 & 25.00 & 0.94438 & 0.04973 & 1 \\
\hline 18 & 100 & 25.00 & 0.89533 & 0.02991 & 1 \\
\hline 19 & 100 & 25.00 & 0.91192 & 0.04269 & 1 \\
\hline 20 & 155 & 54.25 & 0.97540 & 0.01917 & 2 \\
\hline 21 & 155 & 54.25 & 0.97417 & 0.04515 & 3 \\
\hline 22 & 155 & 54.25 & 0.93090 & 0.01240 & 4 \\
\hline 23 & 155 & 54.25 & 0.95978 & 0.03134 & 2 \\
\hline 24 & 197 & 68.95 & 0.92235 & 0.05534 & 3 \\
\hline 25 & 197 & 68.95 & 0.94650 & 0.04933 & 2 \\
\hline 26 & 197 & 68.95 & 0.90208 & 0.05746 & 4 \\
\hline 27 & 197 & 68.95 & 0.95114 & 0.04157 & 3 \\
\hline 28 & 197 & 68.95 & 0.88930 & 0.01559 & 2 \\
\hline 29 & 197 & 68.95 & 0.92506 & 0.02438 & 1 \\
\hline 30 & 350 & 140.00 & 0.97037 & 0.05800 & 3 \\
\hline 31 & 400 & 100.00 & 0.95904 & 0.05735 & 3 \\
\hline 32 & 400 & 100.00 & 0.97438 & 0.03443 & 1 \\
\hline
\end{tabular}

Table 2

LT maintenance decisions for week 19 (case 1).

\begin{tabular}{cl}
\hline Unit no. & Time periods $(12 \mathrm{~h})$ \\
\hline 2 & $254-257$ \\
4 & $253-256$ \\
9 & $262-263$ \\
11 & $258-259$ \\
13 & $262-264$ \\
16 & $265-266$ \\
19 & $262-263$ \\
22 & $261-262$ \\
23 & $253-255$ \\
27 & $256-258$ \\
31 & $259-260$ \\
\hline
\end{tabular}

Table 3

Maintenance and operation costs for GENCO 3 (case 1).

\begin{tabular}{lllcll}
\hline Unit no. & $\begin{array}{l}\text { Maintenance } \\
\text { cost }(€ / \mathrm{MW} / \mathrm{h})\end{array}$ & $\begin{array}{l}\text { Fixed } \\
\text { cost }(€ / \mathrm{h})\end{array}$ & $\begin{array}{l}\text { Production } \\
\text { cost }(€ / \mathrm{MW})\end{array}$ & $\begin{array}{l}\text { Shutdown } \\
\text { cost }(€)\end{array}$ & $\begin{array}{l}\text { Startup } \\
\text { cost }(€)\end{array}$ \\
\hline 12 & - & 211 & 98 & 305 & 284.382 \\
14 & - & 293 & 102 & 201 & 241.2 \\
16 & 45 & 314 & 104 & 205 & 190.65 \\
21 & - & 417 & 94 & 243 & 291.6 \\
24 & - & 372 & 103 & 270 & 232.2 \\
27 & 32 & 463 & 101 & 264 & 348.48 \\
30 & - & 607 & 104 & 316 & 527.72 \\
31 & 55 & 754 & 94 & 408 & 897.6 \\
\hline
\end{tabular}

Each individual GENCO makes reasonable estimates of the generation patterns of rival GENCOs based on the LT maintenance plans released by the ISO and the generators' reliability parameters. The scenarios and their attached weights are generated by 
Table 4

Scenarios for the linear inverse demand function (case 1).

\begin{tabular}{lll}
\hline Elasticity & Linear inverse demand function & Scenarios \\
\hline 0.40 & $P=297.50-0.09239 \cdot Q$ & 1 and 5 \\
0.45 & $P=273.89-0.08213 \cdot Q$ & 2 and 6 \\
0.50 & $P=255.00-0.07391 \cdot Q$ & 3 and 7 \\
0.55 & $P=239.55-0.06719 \cdot Q$ & 4 and 8 \\
\hline
\end{tabular}

the Sequential Monte Carlo (SMC) simulation, which refers to a simulation process over a chronological time span, and is used to simulate the frequency and duration of generators' outages based on the failure rate $(\lambda)$ and the repair rate $(\mu)[27,28]$. The most popular approach to create an artificial system state transition cycle is called state duration sampling, which is based on sampling a probability distribution of generator state duration. The time to failure $\left(t_{F}\right)$ and the time to repair $\left(t_{R}\right)$ are the sampling values of state duration to characterize the availability of a generating unit [25].
In Eqs. (18) and (19) that are used to generate duration sampling, $r_{1}$ and $r_{2}$ are the uniformly distributed random numbers in the interval $[0,1]$

$t_{F}=\frac{-\ln \left(r_{1}\right)}{\lambda}$,

$t_{R}=\frac{-\ln \left(r_{1}\right)}{\mu}$.

\subsection{Scenario reduction}

Scenario reduction techniques are adopted to help the GENCOs in dealing with fewer scenarios, especially when they are asked to submit a limited number of plans for a ST time interval. The main function of scenario reduction techniques is providing a tool for the planners to achieve a reasonably good compromise between computation time and solution accuracy in stochastic optimization

Price $(€)$

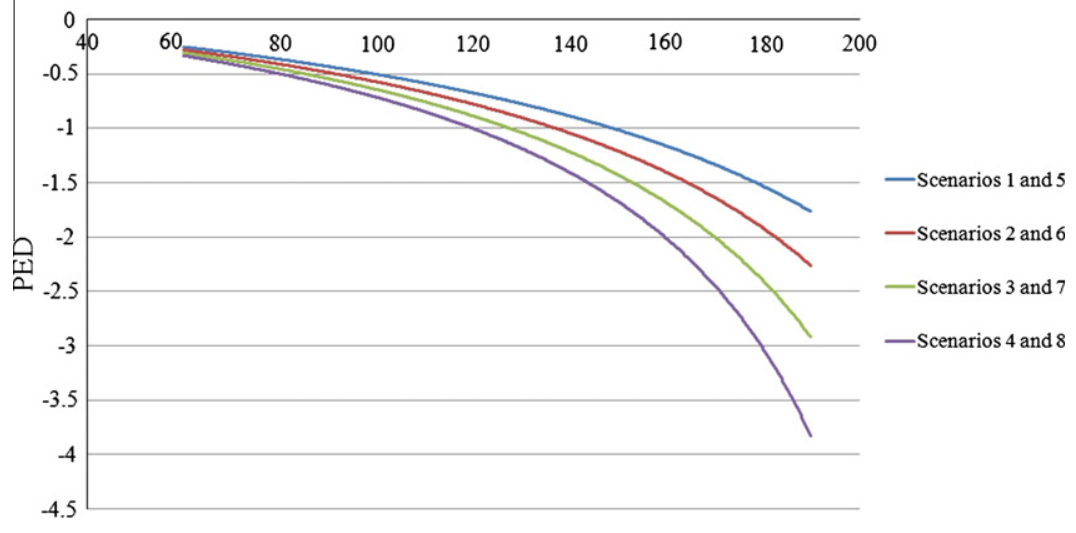

Fig. 2. Scenarios for price elasticity of demand.

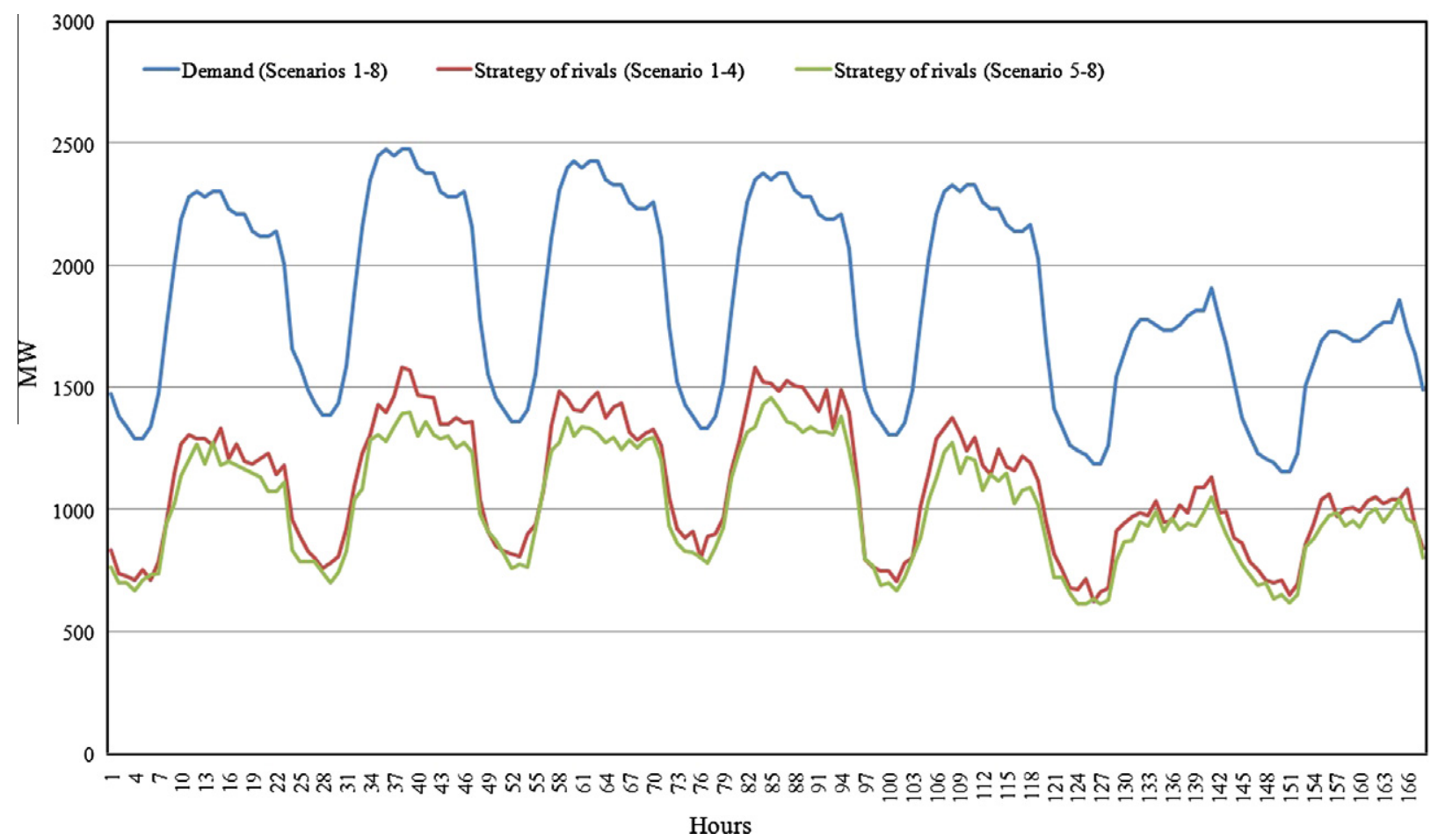

Fig. 3. Scenarios for demand and production patterns of other GENCOs except GENCO 3. 


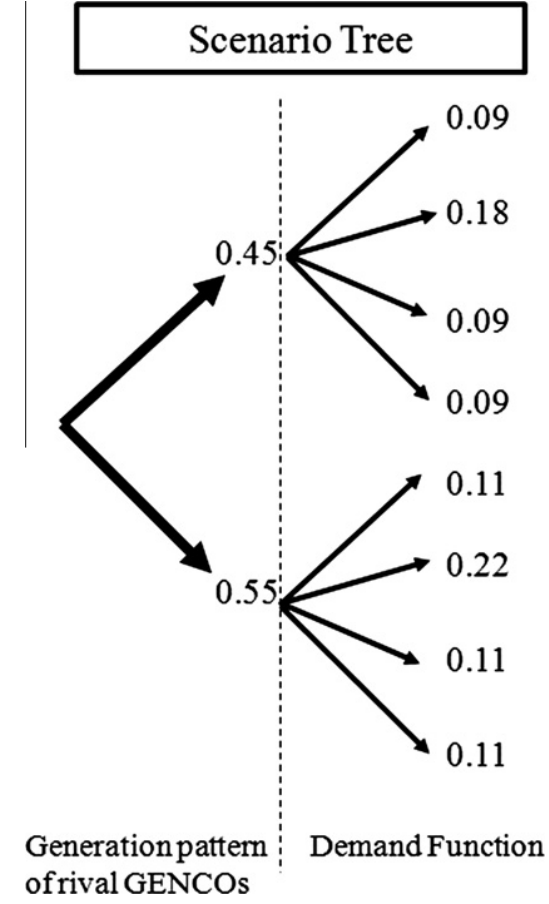

Fig. 4. Representation of uncertainty for each scenario.

models. On the other hand, the ISO asks for a limited number of outage plans and the GENCO has to reduce the number of individual plans that has obtained to submit to the ISO. The reduction is done either after the optimization or before dealing with all the possible scenarios.

Each scenario relates to an individual realization of a multidimensional stochastic process, which is used to deal with the uncertainties of data [29]. The technique proposed in [29] is based on an algorithm that reduces the number of nodes of a set of scenarios without imposing requirements on the stochastic data processes. It bundles close scenarios by measuring the distance between scenarios based on the probability metrics and eliminate scenarios with very low probabilities to determine a limited number of effective scenarios while providing a reasonably good approximation of the system [30]. The new probability of a preserved scenario is equal to the sum of its former probability and the probabilities associated with all deleted scenarios that are closest to it based on the specified probability metrics. The probability of the deleted scenarios is set to zero [31].

The General Algebraic Modeling System (GAMS) provides an efficient tool called SCENRED that implements backward and fast forward reduction methods for scenario reduction and the random data processes [32]. A subset of scenarios with new probabilities is the result of applying scenario reduction approaches. In backward reduction the optimal deletion of a single scenario repeats recursively until a prescribed number of scenarios is deleted [29]. The fast forward algorithm starts with an empty tree and selects the scenarios that minimize the distance function between the reduced and the original tree in an iterative process. The algorithm stops when a specified number of scenarios or a certain distance is reached [16]. Solving the problem for each single scenario and measuring the impact on the objective, and the worst-case scenario probability study are alternative methods that can be used for reducing the number of scenarios [30].

\section{Numerical results}

In case 1, the GENCOs are asked to submit several maintenance outage plans to the ISO for a week ahead without any limitation on the number of plans. Each GENCO considers a specific number of scenarios with regard to the random input parameters, and achieves several distinct outage plans to offer to the ISO. In case 2 , the ISO imposes limitations on the number of proposals that a GENCO can submit. Although the GENCO might encounter many scenarios regarding to the inherent uncertainty in the parameters that it faces during the week ahead, it needs to choose a limited number of them to overcome the computational burden and achieve few plans. Therefore, scenario reduction techniques are implemented to reduce the scenarios and to assign new probabilities to the reduced scenarios.

\subsection{Case 1}

The data of the modified IEEE-RTS test system [3] is used to show the effectiveness of the proposed decision support system during ST maintenance scheduling of GENCOs. In this case, the GENCOs have to submit the maintenance outage plans of a week ahead within 1-h time increments. The participating GENCOs in

Hours

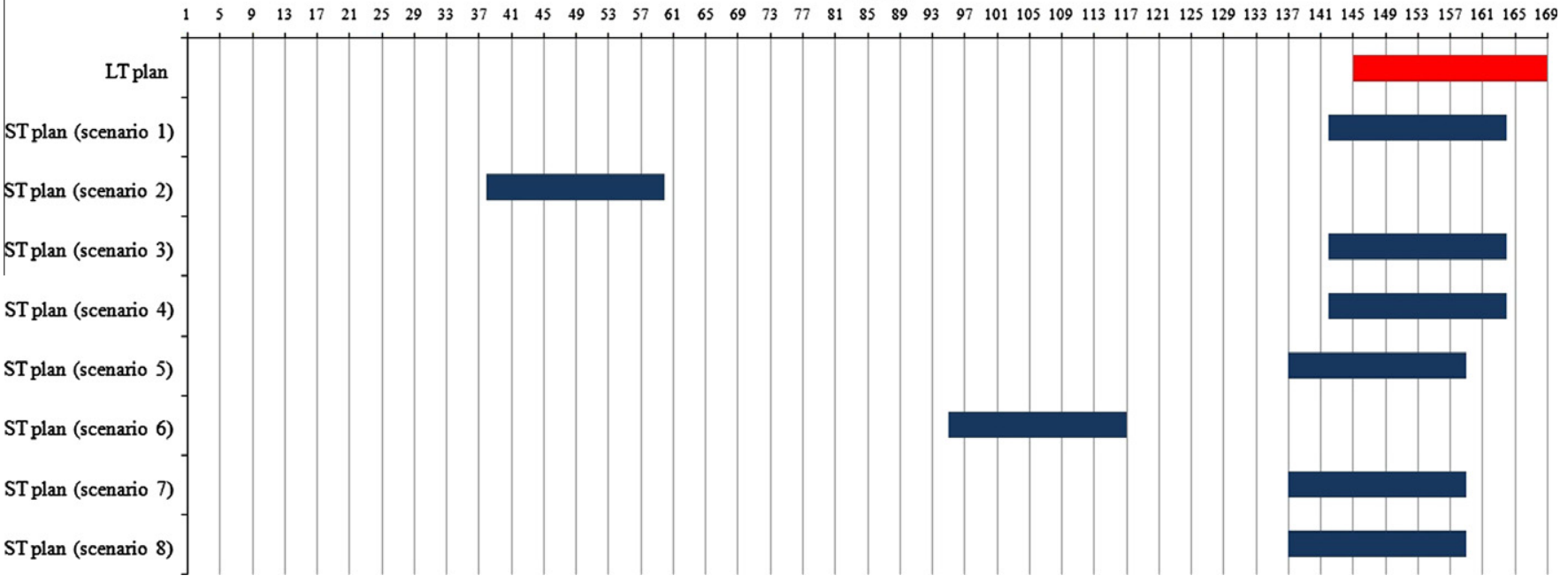

Fig. 5. Weekly maintenance outage scenarios of GENCO 3 for unit 16 . 
Hours

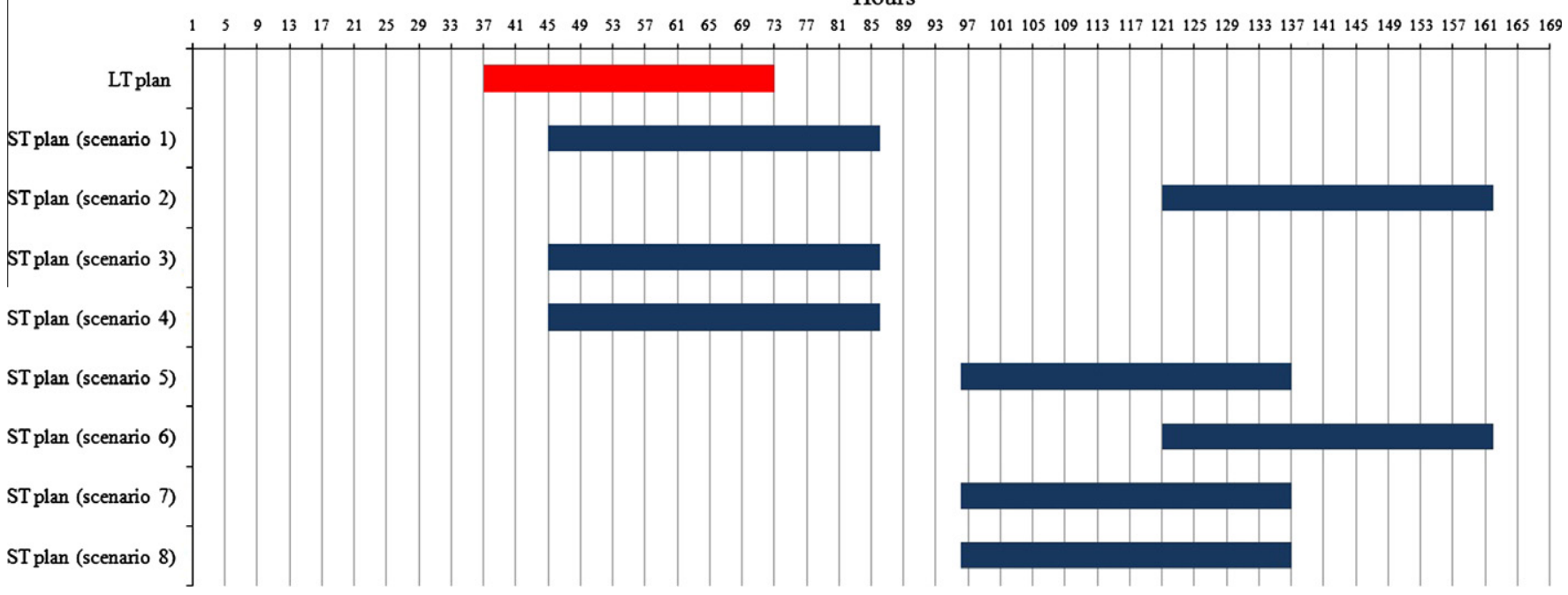

Fig. 6. Weekly maintenance outage scenarios of GENCO 3 for unit 27.

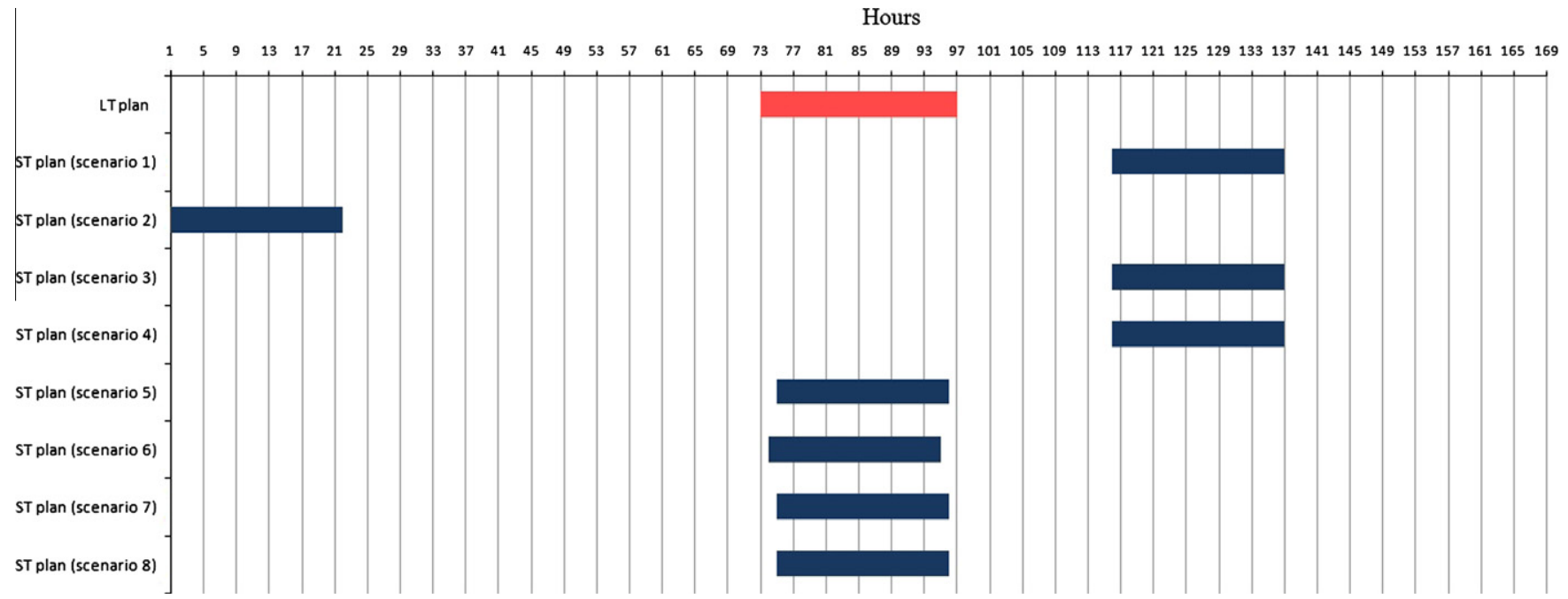

Fig. 7. Weekly maintenance outage scenarios of GENCO 3 for unit 31.

the market have access to the LT maintenance outage plans, which were planned with the time increment of half a day $(12 \mathrm{~h})$. Although the GENCOs are not sure that the rival GENCOs will schedule their units with regard to the LT plans, the best source of information is the LT plans. Table 1 lists the characteristics of the generation sector including the repair and failure rate of the units. To analytically show the effectiveness of the proposed model, GENCO 3 with eight generating units is chosen for the case study.

The ISO asks GENCOs to submit their outage maintenance proposals for week 19 in advance. Maintenance scheduling in the LT time horizon has been released to assist all the participating GENCOs in the market so each GENCO access information on what their rivals might decide. Maintenance outage decisions that were released by the ISO with the time increment of half a day for week 19 , i.e. time periods $253-266$, are shown in Table 2. It is worth mentioning that although these plans were finalized and accepted after several iterations and coordination procedures between the GENCOs and the ISO one or 2 years in advance, there is still the possibility of changing them due to the operational changes that the system and the units have experienced since these plans were finalized.
Units 16, 27 and 31 in Table 2 belong to GENCO 3 and are planned for outage during week 19. However, because of the possible changes in the operational situation of the system, this individual GENCO, like other GENCOs, can submit new plans with different levels of priority to the ISO. Normally, these ST maintenance outage plans are scheduled with the accuracy of $1 \mathrm{~h}$ and give GENCOs more flexibility to locate the outages of their units for the specific time horizon. In ST scheduling, GENCO 3 chooses its maintenance outage plan according to the new needs for maintenance. In week 19, units 16,27 and 31 need 22,41 and 21 h of maintenance respectively. The crew limitation imposes the constraint that only one of the units of GENCO 3 can be considered for maintenance at each period. Table 3 shows maintenance and operation cost for each of the units.

In this case, the scenarios for demand, demand function and the GENCO $i$ 's approximation of the rival GENCOs' generation pattern are available as input data. A series of eight scenarios with different probabilities is considered to model the stochastic scheduling of GENCO 3. The number of scenarios is small and thus no scenario reduction is needed [25]. In Table 4 four different scenarios for the linear inverse demand function with the occurrence probability 
of 0.4 for the elasticity value of 0.45 at price $85 € / \mathrm{MW} \mathrm{h}$, and the probability of 0.2 for other values of elasticity is considered. In these scenarios, as shown in Fig. 2, for low prices, changes in price have a relatively small effect on the electricity demand. However, for high prices (e.g., more than $120 € / M W$ h for scenarios 4 and 8 or $149 € / \mathrm{MW} h$ for scenarios 1 and 5) the electricity demand is relatively elastic. GENCO 3 has two distinct expectations of what other units might produce during the next $168 \mathrm{~h}$ based on LT maintenance scheduling decisions (Fig. 3). As shown in Fig. 3 only one scenario for the demand is considered for week 19 [3].

The probabilities assigned to each of the eight generated scenarios are shown in Fig. 4. In this figure, the branches are different realizations of the random variable [25]. The uncertainty is modeled through a scenario tree (Fig. 4) built as follows [17]:

(1) Two scenarios are generated for the generation pattern of rival GENCOs during the following week.

(2) One scenario is considered for the demand during the week ahead.

(3) Four scenarios are generated model the demand function of loads during the following week based on different possibilities of PED.

The mixed integer linear problem is implemented in GAMS, using the solver CPLEX. The objective function of the stochastic optimization problem regarding to the eight scenarios is 987,492 $€$ with an execution time of 19 min on a machine equipped with two Xeon X5450 processors, each with 4 cores, clocking at 3.0 GHZ with 4 GB of RAM. In Figs. 5-7 the LT maintenance plan and

Table 5

Generating units data (case 2).

\begin{tabular}{|c|c|c|c|c|c|}
\hline $\begin{array}{l}\text { Unit } \\
\text { no. }\end{array}$ & $\begin{array}{l}\text { Capacity } \\
(\mathrm{MW})\end{array}$ & $\begin{array}{l}\text { Minimum } \\
\text { power (MW) }\end{array}$ & $\begin{array}{l}\text { GENCO } \\
\text { No }\end{array}$ & $\begin{array}{l}\text { Mean up } \\
\text { time }(\mathrm{h})\end{array}$ & $\begin{array}{l}\text { Mean down } \\
\text { time }(\mathrm{h})\end{array}$ \\
\hline 1 & 12 & 2.40 & 1 & 56.5 & 1.2 \\
\hline 2 & 20 & 4.00 & 1 & 8.7 & 1.0 \\
\hline 3 & 20 & 4.00 & 3 & 8.7 & 1.0 \\
\hline 4 & 20 & 4.00 & 4 & 8.7 & 1.0 \\
\hline 5 & 20 & 4.00 & 2 & 8.7 & 1.0 \\
\hline 6 & 20 & 4.00 & 4 & 8.7 & 1.0 \\
\hline 7 & 20 & 4.00 & 3 & 8.7 & 1.0 \\
\hline 8 & 76 & 15.20 & 1 & 37.7 & 0.8 \\
\hline 9 & 76 & 15.20 & 4 & 37.7 & 0.8 \\
\hline 10 & 76 & 15.20 & 3 & 37.7 & 0.8 \\
\hline 11 & 76 & 15.20 & 1 & 37.7 & 0.8 \\
\hline 12 & 100 & 25.00 & 2 & 23.1 & 1.0 \\
\hline 13 & 100 & 25.00 & 4 & 23.1 & 1.0 \\
\hline 14 & 100 & 25.00 & 2 & 23.1 & 1.0 \\
\hline 15 & 100 & 25.00 & 4 & 23.1 & 1.0 \\
\hline 16 & 100 & 25.00 & 5 & 23.1 & 1.0 \\
\hline 17 & 100 & 25.00 & 1 & 23.1 & 1.0 \\
\hline 18 & 100 & 25.00 & 2 & 23.1 & 1.0 \\
\hline 19 & 155 & 54.25 & 3 & 18.5 & 0.8 \\
\hline 20 & 155 & 54.25 & 5 & 18.5 & 0.8 \\
\hline 21 & 155 & 54.25 & 2 & 18.5 & 0.8 \\
\hline 22 & 197 & 68.95 & 3 & 18.3 & 1.0 \\
\hline 23 & 197 & 68.95 & 4 & 18.3 & 1.0 \\
\hline 24 & 197 & 68.95 & 5 & 18.3 & 1.0 \\
\hline 25 & 197 & 68.95 & 1 & 18.3 & 1.0 \\
\hline 26 & 197 & 68.95 & 4 & 18.3 & 1.0 \\
\hline 27 & 197 & 68.95 & 3 & 18.3 & 1.0 \\
\hline 28 & 197 & 68.95 & 1 & 18.3 & 1.0 \\
\hline 29 & 350 & 140.00 & 4 & 22.1 & 1.9 \\
\hline 30 & 350 & 140.00 & 2 & 22.1 & 1.9 \\
\hline 31 & 350 & 140.00 & 1 & 22.1 & 1.9 \\
\hline 32 & 350 & 140.00 & 2 & 22.1 & 1.9 \\
\hline 33 & 400 & 100.00 & 3 & 21.2 & 2.9 \\
\hline 34 & 400 & 100.00 & 1 & 21.2 & 2.9 \\
\hline 35 & 400 & 100.00 & 5 & 21.2 & 2.9 \\
\hline 36 & 400 & 100.00 & 4 & 21.2 & 2.9 \\
\hline 37 & 400 & 100.00 & 3 & 21.2 & 2.9 \\
\hline
\end{tabular}

Table 6

LT maintenance decisions for the target week (case 2).

\begin{tabular}{cl}
\hline Unit no. & Time periods $(12 \mathrm{~h})$ \\
\hline 2 & $13-24$ \\
4 & $61-84$ \\
8 & $85-108$ \\
11 & $49-84$ \\
14 & $73-108$ \\
22 & $1-48$ \\
27 & $13-36$ \\
32 & $109-168$ \\
35 & $25-72$
\end{tabular}

Table 7

Daily peak load in percent of weekly peak.

\begin{tabular}{lrlr}
\hline Probability $=0.6$ & \multicolumn{3}{c}{ Probability $=0.4$} \\
\hline Monday & 93 & Monday & 98 \\
Tuesday & 100 & Tuesday & 95 \\
Wednesday & 98 & Wednesday & 100 \\
Thursday & 96 & Thursday & 96 \\
Friday & 94 & Friday & 93 \\
Saturday & 77 & Saturday & 81 \\
Sunday & 75 & Sunday & 76 \\
Scenarios $1,2,5,6,9,10$ & & Scenarios 3, 4, 7, 8, 11, 12 \\
\hline
\end{tabular}

the 8 scenarios for the ST maintenance outage scheduling of units 16,27 and 31 are shown respectively.

The optimal objective function value of the modified stochastic problem is $938,768.4 €$, and consequently the value of $\mathrm{VSS}_{\mathrm{MAX}}$ is $48,723.6 €$.

\subsection{Case 2}

The reliability data of the modified IEEE118-bus test system introduced in $[3,18]$ is used to show how GENCO 4 with nine generating units has to deal with the uncertainties in the system and achieves the final maintenance outage plans that the ISO requests (Table 5). The computational time reduces if the scenario reduction techniques are implemented before running the optimization problem in comparison to deducing the two plans from the result of several maintenance plans achieved after considering all scenarios in the optimization problem.

The outage plan for this time interval that is the result of interaction and coordination between the GENCOs and the ISO in LT maintenance scheduling is shown in Table 6. The ISO provides the opportunity of modifying the plans for those units that are planned for LT maintenance in the target week before the week starts.

The annual peak load of the system is $5500 \mathrm{MW}$ [19], and GENCO 4 considers a finite and discrete set of possible scenarios for the demand in the following week. It is assumed that the weekly peak load in percent of the annual peak is 88.7 for scenarios $1-4,90$ for scenarios 5-8 and 86.1 for scenarios 9-12 with respectively 0.4 , 0.3 and 0.3 as their associated probability of occurrence. The daily peak load in percent of the weekly peak is shown in Table 7. As indicated in Fig. 8 hourly peak load in percent of the daily peak is considered in two distinct patterns, each with different realization for weekdays and weekends. In Table 8 the probability of occurrence for each scenario is shown, based on the values obtained from the aforementioned percentages of the annual peak load. In this case, the stochastic hourly demand is considered as the main source of uncertainty from the viewpoint of the GENCO and other parameters such as the generation estimation of other GENCOs during the 


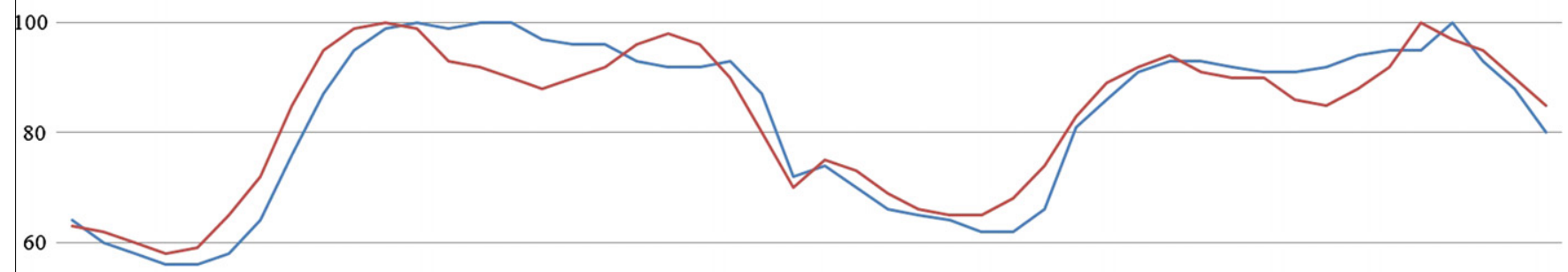

40

20

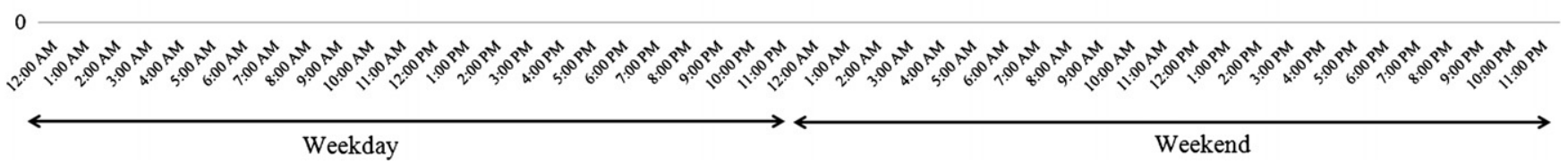

Fig. 8. Hourly peak load in percent of the daily peak (case 2).

Table 8

Probability of each scenario (case 2).

\begin{tabular}{lllllll}
\hline Scenario & 1 & 2 & 3 & 4 & 5 & 6 \\
\hline Probability & 0.12 & 0.12 & 0.08 & 0.08 & 0.09 & 0.09 \\
Scenario & 7 & 8 & 9 & 10 & 11 & 12 \\
Probability & 0.06 & 0.06 & 0.09 & 0.09 & 0.06 & 0.06 \\
\hline
\end{tabular}

Table 9

Scenario reduction for two preserved scenarios.

\begin{tabular}{llll}
\hline & $\begin{array}{l}\text { Preserved } \\
\text { Scenarios }\end{array}$ & $\begin{array}{l}\text { New } \\
\text { probability }\end{array}$ & $\begin{array}{l}\text { Expected } \\
\text { Payoff }(€)\end{array}$ \\
\hline Fast backward method & 1 & 0.5 & $1,059,123.054$ \\
& 2 & 0.5 & \\
Mix of fast backward/ & 1 & 0.5 & $1,059,379.174$ \\
forward methods & 6 & 0.5 & \\
Mix of fast backward/ & 1 & 0.5 & $1,064,507.108$ \\
backward methods & 2 & 0.5 & \\
\hline
\end{tabular}

Table 10

Scenario reduction for three preserved scenarios.

\begin{tabular}{lcll}
\hline & $\begin{array}{c}\text { Preserved } \\
\text { Scenarios }\end{array}$ & $\begin{array}{l}\text { New } \\
\text { probability }\end{array}$ & $\begin{array}{l}\text { Expected } \\
\text { Payoff }(€)\end{array}$ \\
\hline Fast backward method & 1 & 0.5 & $975,963.022$ \\
& 2 & 0.41 & \\
Mix of fast backward/ & 10 & 0.09 & \\
forward methods & 1 & 0.5 & $968,928.677$ \\
& 6 & 0.41 & \\
Mix of fast backward/ & 10 & 0.09 & \\
backward methods & 1 & 0.5 & $993,707.167$ \\
& 2 & 0.41 & \\
\hline
\end{tabular}

Table 11

Scenario reduction for four preserved scenarios.

\begin{tabular}{lcll}
\hline & $\begin{array}{l}\text { Preserved } \\
\text { Scenarios }\end{array}$ & $\begin{array}{l}\text { New } \\
\text { probability }\end{array}$ & $\begin{array}{l}\text { Expected } \\
\text { Payoff }(€)\end{array}$ \\
\hline Fast backward method & 1 & 0.5 & $1,003,766.292$ \\
& 2 & 0.21 & \\
Mix of fast backward/ & 4 & 0.2 & \\
forward methods & 10 & 0.09 & $929,880.712$ \\
& 1 & 0.5 & \\
Mix of fast backward/ & 4 & 0.2 & \\
backward methods & 6 & 0.21 & $1,003,766.292$ \\
& 10 & 0.09 & \\
& 2 & 0.5 & \\
& 4 & 0.21 & \\
& 10 & 0.2 & \\
\hline
\end{tabular}

following week or the parameters of the inverse linear demand function are only demonstrated in one realization.

In ST scheduling, there is not enough time for several data exchange between the GENCOs and the ISO; and accordingly a single stage decision-making structure can be suitable for this environment. The proposed plan provides a basis for ST maintenance scheduling which can be applied jointly with the plan proposed in [6]. In a single stage scheduling, the ISO can accept a limited number of plans from the GENCOs to see if they meet the reliability and security constraints. On the other hand, the GENCOs have various uncertain realizations of the stochastic parameters in the system. In case study 2 , it is assumed that the ISO accepts 2-4 distinct plans from the GENCOs for the following week. The GENCO benefits from the scenario reduction techniques in two ways: by reducing the execution time and finding the most probable scenarios with their new probabilities. SCENRED/GAMS is used to reduce the number of scenarios and the solver CPLEX is used to solve the problem in each situation. Several realizations for the desired number of preserved scenarios and the reduction methods implemented are considered to show the result of having limited 
number of scenarios. The preserved scenarios and the expected payoff when GENCO 4 intends to have 2,3, or 4 preserved scenarios is respectively shown in Tables 9-11.

In ST decision-making for outage periods, the final responsibility of the GENCO is to conclude with specific distinct maintenance plans in order to submit to the ISO. The limit is set by the ISO and the GENCO should just be aware to consider these limitations in maintenance scheduling. The above tables show how the GENCO can deal with various numbers of scenarios, which is the result of stochastic input data. The GENCO should decide which plans to submit to the ISO and it needs to decide how much to pay to the ISO as penalty in a case that the plans deteriorate the system's reliability and security indices.

\section{Conclusions and future work}

The research work presented in this paper characterizes the impact of LT plans on ST maintenance decisions of GENCOs by applying the Cournot model, which has been used for strategic generation dispatch of generating units in electricity markets. The merit of this work is featured by considering the strategic interaction between the GENCOs and including the uncertainty of estimations of each individual GENCO. Test results show how GENCOs accordingly react to the power systems stochastic operation and determine the ST outage schedules based on their estimates of competing GENCOs' generation strategy. The achieved plans are submitted to the ISO, and needs approval before implementation.

Although the GENCOs are not obligated to meet the demand of customers in the maintenance scheduling of their generation assets, including the most probable generation pattern of rival GEN$\mathrm{COs}$ in addition to the forecasted demand curve in the ST time interval provides more realistic assessment of the system. Applying the proposed method by each GENCO in determining the outage decisions increases the chance of acceptance by ISO in comparison to the plans that are achieved without considering the reserve constraints.

The general decision of GENCOs during conventional pricebased maintenance scheduling is to experience the outage of their generating units during low electricity price periods. Thus, the ISO hardly accepts the suggested plans due to probable reliability and security deteriorations in the grid and asks them to resubmit the alternative plans. In the proposed framework, the GENCOs consider the reserve constraints during maintenance outage scheduling and eliminate the need for several data exchange between the GENCOs and the ISO.

Intermittent renewable resources are the main sources causing uncertainty in ST and LT operation scheduling of the power systems with large-scale integration of these units. In the future works, the uncertainties of intermittent renewable resources will be included in decision support systems of GENCOs and the ISO for maintenance scheduling. Transmission maintenance scheduling affects the generation maintenance scheduling, and causes complexity because of changing the system configuration during their maintenance window. In the future research works, planned outages of the transmission sector will be included in the ST maintenance scheduling.

\section{Acknowledgements}

This work is supported by FEDER Funds through COMPETE program and by National Funds through FCT under the Projects FCOMP-01-0124-FEDER: PEst-OE/EEI/UI0760/2011, PTDC/EEAEEL/099832/2008, and PTDC/SEN-ENR/099844/2008.

\section{References}

[1] Nabona N, Pagès A. A three-stage short-term electric power planning procedure for a generation company in a liberalized market. Int J Electric Pow Energy Syst 2007;29:408-21.

[2] Marwali MKC, Shahidehpour SM. Coordination between long-term and shortterm generation scheduling with network constraints. IEEE Trans Pow Syst 2000; $15: 1161-7$

[3] Shahidehpour M, Marwali M. Maintenance scheduling in restructured power systems. Boston (Mass): Kluwer Academic Publishers; 2000.

[4] Ladjici AA, Boudour M. Nash-Cournot equilibrium of a deregulated electricity market using competitive coevolutionary algorithms. Electric Power Syst Res 2011;81:958-66.

[5] Conejo AJ, Garcia-Bertrand R, Diaz-Salazar M. Generation maintenance scheduling in restructured power systems. IEEE Trans Pow Syst 2005;20:984-92.

[6] Fotouhi Ghazvini MA, Morais H, Vale Z. Coordination between mid-term maintenance outage decisions and short-term security-constrained scheduling in smart distribution systems. Appl Energy 2012;96:281-91.

[7] Fotouhi Ghazvini MA, Estebsari A. Market power appearance through game theoretic maintenance scheduling of distributed generations. J Int Rev Electric Eng (IREE) 2011;6:2596-603.

[8] Fotouhi Ghazvini MA, Morais H, Vale Z. Multi-criteria short-term maintenance outage scheduling in smart distribution systems. CIRED 2012 workshop, Lisbon: Integration of Renewables into the Distribution Grid; 2012.

[9] Valenzuela J, Mazumdar M. Cournot prices considering generator availability and demand uncertainty. IEEE Trans Pow Syst 2007;22:116-25.

[10] Zhang SX, Chung CY, Wong KP, Chen H. Analyzing two-settlement electricity market equilibrium by coevolutionary computation approach. IEEE Trans Pow Syst 2009;24:1155-64.

[11] Youfei L, Wu FF. Generator bidding in oligopolistic electricity markets using optimal control: fundamentals and application. IEEE Trans Pow Syst 2006;21:1050-61.

[12] Faria P, Vale Z. Demand response in electrical energy supply: an optimal real time pricing approach. Energy 2011;36:5374-84.

[13] Lei W, Shahidehpour M, Tao L. GENCO's risk-based maintenance outage scheduling. IEEE Trans Pow Syst 2008;23:127-36.

[14] Sahin C, Shahidehpour M, Erkmen I. Generation risk assessment in volatile conditions with wind, hydro, and natural gas units. Appl Energy 2012;96:4-11.

[15] Georgopoulou CA, Giannakoglou KC. Metamodel-assisted evolutionary algorithms for the unit commitment problem with probabilistic outages. Appl Energy 2010;87:1782-92.

[16] Carrion M, Philpott AB, Conejo AJ, Arroyo JM. A stochastic programming approach to electric energy procurement for large consumers. IEEE Trans Pow Syst 2007;22:744-54.

[17] Pousinho HMI, Mendes VMF, Catalão JPS. A risk-averse optimization model for trading wind energy in a market environment under uncertainty. Energy 2011;36:4935-42.

[18] Lei W, Shahidehpour M, Tao L. Stochastic security-constrained unit commitment. IEEE Trans Pow Syst 2007;22:800-11.

[19] Lotfjou A, Shahidehpour M, Yong F, Zuyi L. Security-constrained unit commitment with AC/DC transmission systems. IEEE Trans Pow Syst 2010;25:531-42.

[20] Wang J, Botterud A, Bessa R, Keko H, Carvalho L, Issicaba D, et al. Wind power forecasting uncertainty and unit commitment. Appl Energy 2011;88:4014-23.

[21] Varkani AK, Daraeepour A, Monsef H. A new self-scheduling strategy for integrated operation of wind and pumped-storage power plants in power markets. Appl Energy 2011;88:5002-12.

[22] Samuelson W, Marks SG. Managerial economics. 4th ed. Hoboken (NJ): Wiley; 2003 [Great Britain].

[23] Borenstein S, Bushnell J, Knittel CR. Market power in electricity markets: beyond concentration measures. Energy J 1999;20:65-88.

[24] Conejo AJ. Decomposition techniques in mathematical programming: engineering and science applications. Berlin; London: Springer; 2006.

[25] Conejo AJ, Carrión M, Morales JM. Decision making under uncertainty in electricity markets. New York: Springer; 2010.

[26] Amjady N, Aghaei J, Shayanfar HA. Stochastic multiobjective market clearing of joint energy and reserves auctions ensuring power system security. IEEE Trans Pow Syst 2009;24:1841-54.

[27] Li W. Risk assessment of power systems: models, methods, and applications. Hoboken (NJ): Wiley; 2005 [Great Britain].

[28] Billinton R, Li W. Reliability assessment of electric power systems using Monte Carlo methods. New York (London): Plenum Press; 1994.

[29] Growe-Kuska N, Heitsch H, Romisch W. Scenario reduction and scenario tree construction for power management problems. In: Power Tech Conference Proceedings, 2003 IEEE Bologna, vol. 3; 2003. p. 7.

[30] Lei W, Shahidehpour M, Zuyi L. Comparison of scenario-based and interval optimization approaches to stochastic SCUC. IEEE Trans Pow Syst 2012;27: 913-21.

[31] Parvania M, Fotuhi-Firuzabad M. Demand response scheduling by stochastic SCUC. IEEE Trans Smart Grid 2010;1:89-98.

[32] Scenario reduction module of the GAMS software (SCENRED). <wwwgamscom>. 